\title{
ГИПЕРКОМПЛЕКСНЫЕ МОДЕЛИ МНОГОКАНАЛЬНЫХ ИЗОБРАЖЕНИЙ ${ }^{1}$
}

\author{
В. Г. Лабунец
}

\begin{abstract}
Мы предлагаем новый теоретический подход для обработки многомерных и многокомпонентных изображений, основанный на теории коммутативных гиперкомплексных алгебр, обобщающих алгебру комплексных чисел. Главная цель работы - показать, что коммутативные гиперкомплексные числа могут быть использованы при обработке многоканальных изображений в естественной и эффективной манере. Мы предполагаем, что мозг животных оперирует гиперкомплексными числами, когда обрабатывает многоканальные изображения, которые возникают на ретине глаза. В нашем подходе каждый многоканальный пиксель рассматривается не как K-мерный (K-D) вектор, а как K-D гиперкомплексное число, где K число различных оптических каналов. Это создает эффективную математическую основу для различных функционально-числовых преобразований многоканальных изображений и инвариантного распознавания образов.
\end{abstract}

Ключевые слова: многоканальные изображения, гиперкомплексные алгебры, обработка изображений.

\section{G. Labunets. Hypercomplex models of multichannel images.}

We present a new theoretical approach to the processing of multidimensional and multicomponent images based on the theory of commutative hypercomplex algebras, which generalize the algebra of complex numbers. The main goal of the paper is to show that commutative hypercomplex numbers can be used in multichannel image processing in a natural and effective manner. We suppose that animal brain operates hypercomplex numbers when processing multichannel retinal images. In our approach, each multichannel pixel is regarded as a $K-D$ hypercomplex number rather than a $K-D$ vector, where $K$ is the number of different optical channels. This creates an effective mathematical basis for various function-number transformations of multichannel images and invariant pattern recognition.

Keywords: multichannel images, hypercomplex algebra, image processing.

MSC: 41A45, 42B05, 35S05, 58J40

DOI: $10.21538 / 0134-4889-2020-26-3-69-83$

Посвящается математику, философу и поэту Владимиру Даниловичу Мазурову в связи с его восьмидесятилетием.

\section{Введение}

Многоканальные изображения нашли широкое применение в системах дистанционного зондирования Земли при решении различных научных и прикладных задач (см. [1-4]). В этой работе мы предлагаем новые модели многоканальных изображений с использованием коммутативных гиперкомплексных алгебр. Термин "многоканальные" изображения используется для обозначения изображений с более чем одной компонентой. Они формируются из ряда изображений $f_{\lambda_{0}}(\mathbf{x}), f_{\lambda_{1}}(\mathbf{x}), \ldots, f_{\lambda_{K-1}}(\mathbf{x})$, полученных в различных оптических диапазонах с длинами волн $\lambda_{0}, \lambda_{1}, \ldots, \lambda_{K-1}$, называемых спектральными каналами, где $K$ - число различных оптических каналов. Простым примером является цветное изображение $\mathbf{f}_{C o l}(x, y)=$ $\left(f_{R}(x, y), f_{G}(x, y), f_{B}(x, y)\right)$ с красной $f_{R}(x, y)$, зеленой $f_{G}(x, y)$ и голубой $f_{B}(x, y)$ компонентами. Если изображение собрано из нескольких единиц каналов (меньше, чем 10), то оно называется мультиспектральным, в то время как изображение, состоящее из нескольких десятков

\footnotetext{
${ }^{1}$ Работа выполнена при финансовой поддержке гранта РФФИ № 19-29-09022 19.
} 
или сотен каналов, называется гиперспектральным (конечно, это деление носит условный характер). Многоканальные изображения рассматриваются как $n$ - $D K$-компонентные (векторнозначные) сигналы

$$
\mathbf{f}(\mathbf{x})=\left(f_{0}(\mathbf{x}), f_{1}(\mathbf{x}), \ldots, f_{K-1}(\mathbf{x})\right): \mathbf{R}^{n} \rightarrow \mathbf{V}^{K}
$$

со значениями, лежащими в $K$-D перцептуальном векторном пространстве $\mathbf{V}^{K}$, где $\mathbf{x} \in \mathbf{R}^{n}, n=$ $2,3, \ldots$ Следующие случаи наиболее интересны:

1) 2-D и 3-D бихроматические изображения

$$
\mathbf{f}(\mathbf{x})=\left(f_{0}\left(x_{1}, x_{2}\right), f_{1}\left(x_{1}, x_{2}\right)\right): \mathbf{R}^{2} \rightarrow \mathbf{V}^{2}, \quad \mathbf{f}(\mathbf{x})=\left(f_{0}\left(x_{1}, x_{2}, x_{3}\right), f_{1}\left(x_{1}, x_{2}, x_{3}\right)\right): \mathbf{R}^{3} \rightarrow \mathbf{V}^{2}
$$

2) 2-D и 3-D трихроматические (цветные) изображения

$$
\begin{gathered}
\mathbf{f}(\mathbf{x})=\left(f_{0}\left(x_{1}, x_{2}\right), f_{1}\left(x_{1}, x_{2}\right), f_{2}\left(x_{1}, x_{2}\right)\right): \mathbf{R}^{2} \rightarrow \mathbf{V}_{r g b}^{3}, \\
\mathbf{f}(\mathbf{x})=\left(f_{0}\left(x_{1}, x_{2}, x_{3}\right), f_{1}\left(x_{1}, x_{2}, x_{3}\right), f_{2}\left(x_{1}, x_{2}, x_{3}\right)\right): \mathbf{R}^{3} \rightarrow \mathbf{V}_{r g b}^{3} ;
\end{gathered}
$$

3) 2-D и 3-D K-канальные изображения

$$
\begin{gathered}
\mathbf{f}(\mathbf{x})=\left(f_{0}\left(x_{1}, x_{2}\right), f_{1}\left(x_{1}, x_{2}\right), f_{2}\left(x_{1}, x_{2}\right)\right): \mathbf{R}^{2} \rightarrow \mathbf{V}_{r g b}^{3} \\
\mathbf{f}(\mathbf{x})=\left(f_{0}\left(x_{1}, x_{2}, x_{3}\right), f_{1}\left(x_{1}, x_{2}, x_{3}\right), f_{2}\left(x_{1}, x_{2}, x_{3}\right)\right): \mathbf{R}^{3} \rightarrow \mathbf{V}_{r g b}^{3}
\end{gathered}
$$

3) 2-D и 3-D бихроматические бинокулярные (двухкамерные) изображения (см. [5;6])

$$
\begin{gathered}
\mathbf{f}^{\operatorname{Bin}}\left(x_{1}, x_{2}\right)=\left(\mathbf{f}^{L}(\mathbf{x}), \mathbf{f}^{R}(\mathbf{x})\right): \mathbf{R}^{2} \rightarrow{ }^{L} \mathbf{V}^{2} \oplus^{R} \mathbf{V}^{2}, \\
\mathbf{f}^{\operatorname{Bin}}\left(x_{1}, x_{2}, x_{3}\right)=\left(\mathbf{f}^{L}(\mathbf{x}), \mathbf{f}^{R}(\mathbf{x})\right): \mathbf{R}^{3} \rightarrow{ }^{L} \mathbf{V}^{2} \oplus^{R} \mathbf{V}^{2} ;
\end{gathered}
$$

4) 2-D и 3-D трихроматические (цветные) бинокулярные изображения

$$
\begin{gathered}
\mathbf{f}^{B i n}\left(x_{1}, x_{2}\right)=\left(\mathbf{f}^{L}(\mathbf{x}), \mathbf{f}^{R}(\mathbf{x})\right): \mathbf{R}^{2} \rightarrow{ }^{L} \mathbf{V}_{r g b}^{3} \oplus{ }^{R} \mathbf{V}_{r g b}^{3}, \\
\mathbf{f}^{B i n}\left(x_{1}, x_{2}, x_{3}\right)=\left(\mathbf{f}^{L}(\mathbf{x}), \mathbf{f}^{R}(\mathbf{x})\right): \mathbf{R}^{3} \rightarrow{ }^{L} \mathbf{V}_{r g b}^{3} \oplus{ }^{R} \mathbf{V}_{r g b}^{3} ;
\end{gathered}
$$

5) 2-D и 3-D K-канальные бинокулярные изображения (см. [5;6])

$$
\begin{gathered}
\mathbf{f}^{\operatorname{Bin}}\left(x_{1}, x_{2}\right)=\left(\mathbf{f}^{L}(\mathbf{x}), \mathbf{f}^{R}(\mathbf{x})\right): \mathbf{R}^{2} \rightarrow{ }^{L} \mathbf{V}^{K} \oplus{ }^{R} \mathbf{V}^{K}, \\
\mathbf{f}^{\text {Bin }}\left(x_{1}, x_{2}, x_{3}\right)=\left(\mathbf{f}^{L}(\mathbf{x}), \mathbf{f}^{R}(\mathbf{x})\right): \mathbf{R}^{3} \rightarrow{ }^{L} \mathbf{V}^{K} \oplus{ }^{R} \mathbf{V}^{K},
\end{gathered}
$$

где $\mathbf{f}^{L}(\mathbf{x}), \mathbf{f}^{R}(\mathbf{x})$ - изображения, возникающие на ретине левого и правого глаза соответственно.

Для обработки и распознавания изображений мы превращаем перцептуальные пространства $\mathbf{V}^{K}$ в соответствующие гиперкомплексные алгебры (и называем их перцептуальными алгебрами). Мы разрабатываем алгебраические модели для двух уровней мозга (первый уровень - ретина, второй - Visual Cortex (VC)), используя различные гиперкомплексные алгебры: коммутативные - для первого уровня, где идет обработка и преобразование изображения, и некоммутативные - для второго уровня, где происходит их распознавание. Привлечение некоммутативных алгебр связано с тем, что многие геометрические преобразования изображений (совместные растяжения, вращения, аффинные, проективные) принадлежат некоммутативным группам. Оказывается, что каждое такое преобразование можно описать подходящим многомерным гиперкомплексным числом (например, кватернионом для вращения 3-D изображений).

Одна из наших гипотез состоит в том, что мозг животных должен иметь врожденные знания о подобных числах и уметь ими оперировать в режиме распознавания образов. В следующей части работы мы покажем, что алгебраические модели многоканальных изображений 
позволяют разрабатывать простые, наглядные и эффективные (с вычислительной точки зрения) инвариантные алгоритмы распознавания таких изображений с использованием быстрых преобразований Фурье - Клиффорда - Галуа.

В предлагаемом алгебро-геометрическом подходе каждый многоканальный пиксель рассматривается не как $K$-D вектор, а как $K$-D гиперкомплексное число (заметим, что числовая природа не отменяет векторной природы гиперкомплексного числа: просто векторное пространство оснащается операцией векторного умножения векторов, которые интерпретируются как числа).

В этой работе мы интерпретируем многоканальное изображение, возникающее на ретине глаза, как мультиплетно-значный сигнал

$$
\mathbf{f}(\mathbf{x})=\left(f_{0}(\mathbf{x}), f_{1}(\mathbf{x}), \ldots, f_{K-1}(\mathbf{x})\right)=\sum_{s=0}^{K-1} f_{s}(\mathbf{x}) \varepsilon^{s}=f_{0}(\mathbf{x}) \varepsilon^{0}+f_{1}(\mathbf{x}) \varepsilon^{1}+\ldots+f_{K-1}(\mathbf{x}) \varepsilon^{K-1}
$$

который принимает значения в одной из трех коммутативных алгебр

$$
\operatorname{Alg}_{K}^{R e t}\left(\mathbf{R} \mid \varepsilon^{0}, \varepsilon^{1}, \ldots, \varepsilon^{K-1}\right)=\operatorname{Alg}_{K}^{R e t},
$$

где $\varepsilon^{K}=-1, \varepsilon^{K}=0$ или $\varepsilon^{K}=+1$. Здесь $\varepsilon^{0}, \varepsilon^{1}, \ldots, \varepsilon^{K-1}-$ гипермнимые единицы (гиперспектральные единицы) с коммутативным законом умножения

$$
\begin{aligned}
& \varepsilon^{r} \cdot \varepsilon^{s}=\left\{\begin{array}{lll}
\varepsilon^{r \oplus s(\bmod K)}, & \text { если } & \varepsilon^{K}=+1, \\
\operatorname{Hev}(l-m) \varepsilon^{r \oplus s(\bmod K),}, & \text { если } & \varepsilon^{K}=0, \\
\operatorname{Sign}(l-m) \varepsilon^{r \oplus s(\bmod K)}, & \text { если } & \varepsilon^{K}=-1 ;
\end{array}\right. \\
& \operatorname{Sign}(x)=\left\{\begin{array}{rl}
+1, & x \geq 0, \\
-1, & x<0 ;
\end{array} \quad \operatorname{Hev}(x)=\left\{\begin{aligned}
+1, & x \geq 0, \\
0, & x<0,
\end{aligned}\right.\right.
\end{aligned}
$$

где $l \oplus m-$ сложение по модулю $K$, используемые функции - Signum и Heaviside.

Мы опираемся на следующие гипотезы:

1. Мозг интерпретирует каждый пиксель изображения не как многомерный вектор, а как многомерное гиперкомплексное число. Если мы допускаем существование векторной природы пикселей, то можно пойти и дальше: допустить возможность умножения этих векторов. Тем самым мы не опровергаем векторную природу пикселя, а обогащаем ее дополнительными математическими возможностями. Речь идет о расширении возможностей математического языка описания реальной действительности за счет введения операции умножения пикселей.

2. Визуальные системы животных с различной эволюционной историей используют различные гиперкомплексные алгебры для обработки цветных и многоканальных изображений. По-видимому, отдел Visual Cortex обладает способностью оперировать пикселями изображения как с гиперкомплексными числами.

3. Головной мозг использует различные алгебры на двух уровнях: коммутативные алгебры - на уровне ретины при их обработке и некоммутативные алгебры - на уровне VC при распознавании изображений.

Мы знаем, что животные способны почти мгновенно и эффективно распознавать окружающие объекты. Для инженера важно описать это явление на таком математическом языке, который позволил бы ему построить техническую систему, способную работать не менее эффективно, чем исходная биологическая система; вопрос об адекватности используемой математической модели для инженера (в отличие от биолога и физика) стоит на втором плане.

Мы не согласны с Л.Кронекером, утверждавшим, что "Бог создал иелые числа, а все остальное - дело рук человеческих". Мы предполагаем, что Бог был первым инженером, который знал высшую алгебру и использовал теорию гиперкомплексных чисел для проектирования визуальных систем живых существ. 


\section{1. Алгебраические модели серых и бихроматических изображений}

Бихроматические 2-D изображения $\mathbf{f}\left(x_{1}, x_{2}\right)=\left(f_{0}\left(x_{1}, x_{2}\right), f_{1}\left(x_{1}, x_{2}\right)\right): \mathbf{R}^{2} \rightarrow \mathbf{V}^{2}$ обладают двумя атрибутами: $\mathbf{R}^{2}, \mathbf{V}^{2}$ - физическим и визуальным пространствами. Мы оснащаем эти пространства структурами 2-D алгебр обобщенных комплексных чисел $\operatorname{Alg}_{2}^{S p}(\mathbf{R} \mid 1, I)$ и $A l g_{2}^{V i s}(\mathbf{R} \mid 1, J)$ соответственно, т. е.

$$
\begin{gathered}
\mathbf{R}^{2} \rightarrow A l g_{2}^{S p}(\mathbf{R} \mid 1, I):=\mathbf{R}+\mathbf{R} I=\left\{\mathbf{z}=x_{1}+I x_{2} \mid x_{1}, x_{2} \in \mathbf{R}\right\}, \\
\mathbf{V}^{2} \rightarrow A l g_{2}^{V i s}(\mathbf{R} \mid 1, J):=\mathbf{R}+\mathbf{R} J=\{\mathrm{Z}=r+J g \mid r, g \in \mathbf{R}\},
\end{gathered}
$$

где $I-$ пространственная и $J$ - бихроматическая мнимые единицы. Эти алгебры называются пространственной и бихроматической алгебрами (см. [7-9]) физического $\mathbf{R}^{2}$ и визуального (перцептуального) $\mathbf{V}^{2}$ пространств. Существуют три пространственные алгебры.

1. Если $I^{2} \equiv I_{-}^{2}=-1$, то алгебра $A l g_{2}^{S p}\left(\mathbf{R} \mid 1, I_{-}\right)=\left\{\mathbf{z}=x+I_{-} y \mid x, y \in \mathbf{R} ; I_{-}^{2}=-1\right\}$ является полем комплексных пространственных чисел, где $I_{-}=i-$ обычная классическая (эллиптическая) мнимая единица.

2. Если $I^{2} \equiv I_{+}^{2}=+1$, то алгебра $A l g_{2}^{S p}\left(\mathbf{R} \mid 1, I_{+}\right)=\left\{\mathbf{z}=x+I_{+} y \mid x, y \in \mathbf{R} ; I_{+}^{2}=+1\right\}$ является кольцом двоичных пространственных комплексных чисел, где $I_{+}=e-$ классическая двойная (гиперболическая) мнимая единица.

3. Если $I^{2} \equiv I_{0}^{2}=0$, то алгебра $\operatorname{Alg}_{2}^{S p}\left(\mathbf{R} \mid 1, I_{0}\right)=\left\{\mathbf{z}=x+I_{0} y \mid x, y \in \mathbf{R} ; I_{0}^{2}=-1\right\}$ является кольцом дуальных пространственных комплексных чисел, где $I_{0} \equiv \varepsilon-$ классическая дуальная (параболическая) мнимая единица.

Известно (см. [10]), что эти алгебры порождают различные метрики в 2-D физическом пространстве (Евклидову, Минковского и метрику Галилея). Это позволяет на алгебраическом языке просто описывать геометрические преобразования изображений различной геометрической природы.

Используя алгебры $A l g_{2}^{S p}(\mathbf{R} \mid 1, I)$, можно ввести алгебраические модели серых изображений в виде функции одной обобщенной комплексной переменной $\mathbf{f}(x+I y): A l g_{2}^{S p}(\mathbf{R} \mid 1, I) \rightarrow \mathbf{R}$. Существуют три типа таких изображений:

$$
\text { 1) } \left.\left.{ }^{-} \mathbf{f}(\mathbf{z}): A l g_{2}^{S p}\left(\mathbf{R} \mid 1, I_{-}\right) \rightarrow \mathbf{R}, 2\right)^{0} \mathbf{f}(\mathbf{z}): A l g_{2}^{S p}\left(\mathbf{R} \mid 1, I_{0}\right) \rightarrow \mathbf{R}, 3\right)^{+} \mathbf{f}(\mathbf{z}): A l g_{2}^{S p}\left(\mathbf{R} \mid 1, I_{+}\right) \rightarrow \mathbf{R} .
$$

Первые изображения определены на евклидовой (эллиптической) плоскости, вторые - на плоскости Минковского (гиперболической плоскости), третьи - на плоскости Галилея (параболической плоскости).

Аналогично существуют три перцептуальные алгебры с различными геометрическими (метрическими) свойствами.

1. Если $J^{2} \equiv J_{-}^{2}=-1$, то перцептуальная алгебра $A l g_{2}^{\text {Vis }}\left(\mathbf{R} \mid 1, J_{-}\right)=\left\{\mathbf{z}=x+J_{-} y \mid\right.$ $\left.x, y \in \mathbf{R} ; J_{-}^{2}=-1\right\}$ есть поле комплексных бихроматических чисел, где $J_{-}-$бихроматическая мнимая единица, подобная обычной классической мнимой единице $J_{-} \approx i$.

2. Если $J^{2} \equiv J_{+}^{2}=+1$, то перцептуальная алгебра $\operatorname{Alg}_{2}^{V i s}\left(\mathbf{R} \mid 1, J_{+}\right)=\left\{\mathbf{z}=x+J_{+} y \mid\right.$ $\left.x, y \in \mathbf{R} ; J_{+}^{2}=+1\right\}$ есть кольцо двойных бихроматических чисел, где $J_{+}-$бихроматическая мнимая единица, подобная обычной двойной единице $J_{+} \approx e$.

3. Если $J^{2} \equiv J_{0}^{2}=0$, то перцептуальная алгебра $\operatorname{Alg}_{2}^{V i s}\left(\mathbf{R} \mid 1, J_{0}\right)=\left\{\mathbf{z}=x+J_{0} y \mid\right.$ $\left.x, y \in \mathbf{R} ; J_{0}^{2}=0\right\}$ представляет собой кольцо дуальных бихроматических чисел, где $J_{0}-$ бихроматическая мнимая единица, подобная обычной дуальной единице $J_{0} \approx \varepsilon$.

Таким образом, существуют девять моделей бихроматических изображений $\mathbf{f}(\mathbf{z})$ : $A l g_{2}^{S p}(\mathbf{R} \mid 1, I) \rightarrow \operatorname{Alg}_{2}^{V i s}(\mathbf{R} \mid 1, J)$, представленных ниже:

$$
\begin{array}{ll}
{ }^{-,}-{ }^{-} \mathbf{f}(\mathbf{z}): A l g_{2}^{S p}\left(\mathbf{R} \mid 1, I_{-}\right) \rightarrow A l g_{2}^{V i s}\left(\mathbf{R} \mid 1, J_{-}\right), & { }^{-, 0} \mathbf{f}(\mathbf{z}): A l g_{2}^{S p}\left(\mathbf{R} \mid 1, I_{-}\right) \rightarrow A l g_{2}^{V i s}\left(\mathbf{R} \mid 1, J_{0}\right), \\
{ }^{0,} \mathbf{f}(\mathbf{z}): A l g_{2}^{S p}\left(\mathbf{R} \mid 1, I_{0}\right) \rightarrow \operatorname{Alg}{ }_{2}^{V i s}\left(\mathbf{R} \mid 1, J_{-}\right), & { }^{0,0} \mathbf{f}(\mathbf{z}): A l g_{2}^{S p}\left(\mathbf{R} \mid 1, I_{0}\right) \rightarrow A l g_{2}^{V i s}\left(\mathbf{R} \mid 1, J_{0}\right), \\
{ }^{+,}-\mathbf{f}(\mathbf{z}): A l g_{2}^{S p}\left(\mathbf{R} \mid 1, I_{+}\right) \rightarrow A l g_{2}^{V i s}\left(\mathbf{R} \mid 1, J_{-}\right), & { }^{+, 0} \mathbf{f}(\mathbf{z}): A l g_{2}^{S p}\left(\mathbf{R} \mid 1, I_{+}\right) \rightarrow A l g_{2}^{V i s}\left(\mathbf{R} \mid 1, J_{0}\right),
\end{array}
$$




$$
\begin{aligned}
& { }^{-,+} \mathbf{f}(\mathbf{z}): A l g_{2}^{S p}\left(\mathbf{R} \mid 1, I_{-}\right) \rightarrow A l g_{2}^{V i s}\left(\mathbf{R} \mid 1, J_{+}\right), \\
& { }^{0,+} \mathbf{f}(\mathbf{z}): A l g_{2}^{S p}\left(\mathbf{R} \mid 1, I_{0}\right) \rightarrow A l g_{2}^{V i s}\left(\mathbf{R} \mid 1, J_{+}\right), \\
& { }^{+,}+\mathbf{f}(\mathbf{z}): A l g_{2}^{S p}\left(\mathbf{R} \mid 1, I_{+}\right) \rightarrow A l g_{2}^{V i s}\left(\mathbf{R} \mid 1, J_{+}\right) .
\end{aligned}
$$

Для обозначения шести алгебр (трех пространственных и трех перцептуальных) будем пользоваться символом $A l g_{2}^{R e t}(\mathbf{R} \mid 1, B)$ или символом $A l g_{2}^{\text {Ret }}$ для краткости, где $A l g_{2}^{\text {Ret }}(\mathbf{R} \mid 1, B)=$ $A l g_{2}^{S p}(\mathbf{R} \mid 1, I)$, если $B=I$, и $A l g_{2}^{R e t}(\mathbf{R} \mid 1, B)=A l g_{2}^{V i s}(\mathbf{R} \mid 1, J)$, если $B=J$.

В алгебрах $A l g_{2}^{R e t}$ имеет место операция сопряжения, которая отображает каждый элемент $Z=a+B b$ в элемент $\bar{Z}=\overline{a+B b}=a-B b$. Пусть $Z=a+B b$, тогда квадратичная форма $N(Z):=\|Z\|=Z \bar{Z}=a^{2}-B^{2} b^{2}$ называется псевдонормой числа $Z=a+B b$, a $|Z|=\sqrt{N(Z)}=$ $\sqrt{Z \bar{Z}}$ - его модулем. Очевидно, $N\left(Z_{1} Z_{2}\right)=N\left(Z_{1}\right) N\left(Z_{2}\right)$. Поэтому 2-D алгебры $A l g_{2}^{S p} \equiv$ $A l g_{2}^{S p}(\mathbf{R} \mid 1, I)$ и $A l g_{2}^{V i s} \equiv A l g_{2}^{V i s}(\mathbf{R} \mid 1, J)$ превращаются в псевдометрические пространства:

$$
\begin{gathered}
A l g_{2}^{S p}(\mathbf{R} \mid 1, J) \rightarrow \mathbf{G e o}_{2}^{S p\left(s_{1}, s_{2}\right)}=\left\langle\mathbf{R}^{2}, \rho\left(\mathbf{z}_{1}, \mathbf{z}_{2}\right)\right\rangle, \\
A l g_{2}^{V i s}(\mathbf{R} \mid 1, J) \rightarrow \mathbf{G e o}_{2}^{V i s\left(s_{1}, s_{2}\right)}=\left\langle\mathbf{V}^{2}, \rho\left(Z_{1}, Z_{2}\right)\right\rangle,
\end{gathered}
$$

если в них определить следующие псевдометрики:

$$
\rho\left(Z_{1}, Z_{2}\right):=\sqrt{\left(Z_{2}-Z_{1}\right) \overline{\left(Z_{2}-Z_{1}\right)}}= \begin{cases}\sqrt{\left(a_{2}-a_{1}\right)^{2}+\left(b_{2}-b_{1}\right)^{2}}, & Z \in \operatorname{Alg}_{2}^{R e t}\left(\mathbf{R} \mid B_{-}\right), \\ \sqrt{\left(a_{2}-a_{1}\right)^{2}-\left(b_{2}-b_{1}\right)^{2}}, & Z \in A l g_{2}^{R e t}\left(\mathbf{R} \mid B_{+}\right), \\ \left|a_{2}-a_{1}\right|, & Z \in \operatorname{Alg}_{2}^{\operatorname{Ret}}\left(\mathbf{R} \mid B_{0}\right),\end{cases}
$$

где $Z_{1}=a_{1}+B b_{1}, Z_{2}=a_{2}+B b_{2}$, а два символа $\left(s_{1}=+1, s_{2}=-1,0,+1\right)$ в выражениях $\mathbf{G e o}_{2}^{S p\left(s_{1}, s_{2}\right)}, \mathbf{G e o}_{2}^{V i s\left(s_{1}, s_{2}\right)}$ обозначают сигнатуру пространств. В зависимости от сигнатуры $\left(s_{1}, s_{2}\right)$ алгебры трансформируются в следующие псевдометрические пространства $\mathbf{G e o}_{2}^{\operatorname{Ret}\left(s_{1}, s_{2}\right)}$ :

- двухмерную геометрию Евклида $\mathbf{G e o}_{2}^{\operatorname{Ret}(+,+)}=\left\langle A l g_{2}^{\operatorname{Ret}}\left(\mathbf{R} \mid B_{+}\right) ; \rho\right\rangle$ (пространственную $\mathbf{G e o}_{2}^{S p(+,+)}$ и перцептуальную $\left.\mathbf{G e o}_{2}^{V i s(+,+)}\right)$;

- двухмерную геометрию Минковского $\mathbf{G e o}_{2}^{\operatorname{Ret}(+,-)}=\left\langle A l g_{2}^{R e t}\left(\mathbf{R} \mid B_{-}\right) ; \rho\right\rangle$ (пространственную $\mathbf{G e o}_{2}^{S p(+,-)}$ и перцептуальную $\left.\mathbf{G e o}_{2}^{V i s(+,-)}\right)$;

- двухмерную геометрию Галилея $\mathbf{G e o}_{2}^{\operatorname{Ret}(+, 0)}=\left\langle A l g_{2}^{R e t}\left(\mathbf{R} \mid B_{0}\right) ; \rho\right\rangle$ (пространственную $\mathbf{G e o}_{2}^{S p(+, 0)}$ и перцептуальную $\left.\mathbf{G e o}{ }_{2}^{\text {Vis(+, }}{ }^{\text {(t) }}\right)$.

Множество всех точек обобщенной комплексной плоскости $\mathbf{G e o}_{2}^{\operatorname{Ret}\left(s_{1}, s_{2}\right)}$, удовлетворяющее уравнению $|Z|^{2}=a^{2}-B^{2} b^{2}=R^{2}$, называется $\mathbf{G e o}_{2}^{\operatorname{Ret}\left(s_{1}, s_{2}\right)}$-окружсностъю радиусом $R$ с центром в начале координат. Пусть $\operatorname{Alg}_{2}^{R e t}(\mathbf{R} \mid 1, B) \equiv \operatorname{Alg}_{2}^{S p}(\mathbf{R} \mid 1, I)$, тогда имеются три типа окружностей: $\mathbf{G e o}_{2}^{S p(+,+)}$-окружность - классическая (Евклидова окружность), $\mathbf{G e o}_{2}^{S p(+,-)}$ окружность (гиперболическая окружность) - окружность Минковского и $\mathbf{G e o}_{2}^{S p(+, 0)}$-окружность (окружность Галилея) - две параллельные линии.

Пусть $Z=a+B b-$ произвольное обобщенное комплексное число (пространственное или бихроматическое), тогда число $Z_{0}=Z /|Z|$ будет иметь единичный модуль, если $|Z|=R \neq 0$. Легко проверить, что $Z=|Z| \cdot(a /|Z|+B \cdot b /|Z|)=R(\cos \alpha+B \sin \alpha)=R e^{B \theta}$, где $\cos \alpha$ и $\sin \alpha$ суть тригонометрические функции Евклида (классические $\cos \alpha=\cos \alpha, \sin \alpha=\sin \alpha$ ), Минковского (гиперболические $\cos \alpha=\operatorname{ch} \alpha, \sin \alpha=\operatorname{sh} \alpha)$ и Галилея $(\cos \alpha=\operatorname{cg} \alpha, \sin \alpha=$ $\operatorname{sg} \alpha)$.

О п р е д е л е н и е 1. Бихроматическим изображением

$$
\mathbf{f}(\mathbf{z}): \operatorname{Alg}_{2}^{S p}(\mathbf{R} \mid 1, I) \rightarrow A l g_{2}^{V i s}(\mathbf{R} \mid 1, J)
$$


называется $A l g_{2}^{V i s}(\mathbf{R} \mid J)$-значная функция, зависящая от комплексной переменной $\mathbf{z} \in A l g_{2}^{S p}(\mathbf{R} \mid$ $1, I)$ :

$$
\mathbf{f}(\mathbf{z})=f_{0}\left(x_{1}+I x_{2}\right)+J \cdot f_{1}\left(x_{1}+I x_{2}\right) .
$$

О п р е д е л е н и е 2. Преобразования

$$
\mathbf{z}^{\prime}=\mathbf{z}+\mathbf{w}, \quad \mathbf{z}^{\prime}=\lambda \mathbf{z}, \quad \mathbf{z}^{\prime}=e^{I \varphi_{s p}} \mathbf{z} ; \quad Z^{\prime}=Z+W, \quad Z^{\prime}=\mu Z, \quad Z^{\prime}=e^{J \theta_{c h}} Z,
$$

где $\mathbf{z}, \mathbf{z}^{\prime}, \mathbf{w} \in A l g_{2}^{S p}$ и $Z, Z, W \in A l g_{2}^{V i s}$, называются трансляцией, масштабированием и вращением физического $\mathbf{G e o}_{2}^{S p\left(s_{1}, s_{2}\right)}$ и бихроматического $\mathbf{G e o}_{2}^{V i s\left(s_{1}, s_{2}\right)}$ пространств, соответственно.

Эти преобразования формируют: 1) две группы пространственных $\operatorname{Tr}\left(\mathbf{G e o}_{2}^{S p\left(s_{1}, s_{2}\right)}\right)$ и бихроматических $\operatorname{Tr}\left(\mathbf{G e o}_{2}^{V i s\left(s_{1}, s_{2}\right)}\right)$ трансляций; 2) две группы пространственных $\mathbf{S c}\left(\mathbf{G e o}_{2}{ }_{p}\left(s_{1}, s_{2}\right)\right)$ и бихроматических $\mathbf{S c}\left(\mathbf{G e o}_{2}^{\text {Vis }\left(s_{1}, s_{2}\right)}\right)$ масштабных преобразований; 3) две группы физических $\boldsymbol{\operatorname { R o t }}\left(\mathbf{G e o}_{2}^{S p\left(s_{1}, s_{2}\right)}\right)$ и бихроматических $\boldsymbol{\operatorname { R o t }}\left(\mathbf{G e o}_{2} \operatorname{Vis}^{\left(s_{1}, s_{2}\right)}\right)$ вращений.

Преобразования изображений (геометрические и цветовые искажения) в физическом и в перцептуальном пространствах могут быть описаны на языках пространственной и перцептуальной алгебр. Эти искажения могут быть вызваны: 1) пространственными преобразованиями (трансляциями $\mathbf{z}^{\prime}=\mathbf{z}+\mathbf{w}$, вращениями $\mathbf{z}^{\prime}=e^{I \varphi_{s p}} \mathbf{z}$, изменениями масштаба $\mathbf{z}^{\prime}=\lambda \mathbf{z}$ ) и 2) бихроматическими преобразованиями (бихроматической трансляцией $\mathbf{f}+W$, преобразованием

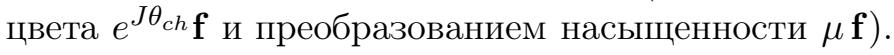

Если $\mathbf{f}(\mathbf{z})$ - некоторое исходное бихроматическое изображение, то изображение

$$
\mu, \theta_{c h}, W \mathbf{f}_{\lambda, \varphi_{s p}, \mathbf{w}}(\mathbf{z})=\mu e^{J \theta_{c h}} \cdot \mathbf{f}\left(\lambda e^{J \varphi_{s p}} \mathbf{z}+\mathbf{w}\right)+W
$$

является его искаженной версией. Пространственные искажения здесь вызваны преобразованиями физического пространства: $\mathbf{z} \rightarrow \lambda e^{J \varphi_{s p}} \mathbf{z}+\mathbf{w}$, а цветовые - преобразованиями перцептуального пространства $\mathbf{f} \rightarrow \mu e^{J \theta_{c h}} \cdot \mathbf{f}+W$.

Оказывается, что алгебраические модели бихроматического изображения (1) и его искаженной версии (2) имеют универсальный характер в том смысле, что многие типы многоканальных изображений, определенные на евклидовых и неевклидовых пространствах, могут быть записаны в аналогичной форме с применением гиперкомплексных алгебр. Это обстоятельство позволяет разрабатывать новые алгоритмы обработки и инвариантного распознавания изображений.

\section{2. Алгебраические модели цветных изображений}

Цветное изображение является векторно-значной функцией вида $\mathbf{f}(\mathbf{x}): \mathbf{R}^{n} \rightarrow \mathbf{V}_{r g b}^{3}$, где $\mathbf{V}_{r g b}^{3}$ - трихроматическое (цветовое) RGB-пространство. Мы будем интерпретировать его как триплетно-значный сигнал $\mathbf{f}(\mathbf{x})=f_{r}(\mathbf{x}) 1+f_{g}(\mathbf{x}) \varepsilon_{c o l}^{1}+f_{b}(\mathbf{x}) \varepsilon_{c o l}^{2}$, который принимает значения в триплетной (цветной) алгебре $A l g_{3}^{V i s}\left(\mathbf{R} \mid 1, \varepsilon, \varepsilon^{2}\right):=\mathbf{R} 1_{c o l}+\mathbf{R} \varepsilon_{c o l}^{1}+\mathbf{R} \varepsilon_{c o l}^{2}$, где $1_{c o l}, \varepsilon_{c o l}^{1}, \varepsilon_{c o l}^{2}-$ три гипермнимые (цветные) единицы с одним из трех свойств: $\varepsilon_{c o l}^{3}=+1, \varepsilon_{c o l}^{3}=0$ либо $\varepsilon_{c o l}^{3}=-1$ (см. [11-16]). Для краткости будем обозначать их как $1, \varepsilon^{1}, \varepsilon^{2}$. Очевидно, существуют три перцептуальные цветные алгебры.

1. Если $\varepsilon^{3}=\varepsilon_{-}^{3}=-1$, то $A l g_{3}^{V i s}\left(\mathbf{R} \mid 1, \varepsilon_{-}, \varepsilon_{-}^{2}\right):=\mathbf{R} 1+\mathbf{R} \varepsilon_{-}^{1}+\mathbf{R} \varepsilon_{-}^{2}=\left\{\mathcal{C}=r 1+g \varepsilon_{-}^{1}+\right.$ $\left.b \varepsilon_{-}^{2} \mid r, g, b \in \mathbf{R}\right\}-$ цветная алгебра цветных ациклических чисел.

2. Если $\varepsilon^{3}=\varepsilon_{+}^{3}=+1$, то $A l g_{3}^{V i s}\left(\mathbf{R} \mid 1, \varepsilon_{+}, \varepsilon_{+}^{2}\right):=\mathbf{R} 1+\mathbf{R} \varepsilon_{+}^{1}+\mathbf{R} \varepsilon_{+}^{2}=\left\{\mathcal{C}=r 1+g \varepsilon_{+}^{1}+\right.$ $\left.b \varepsilon_{+}^{2} \mid r, g, b \in \mathbf{R}\right\}$ - цветная алгебра цветных циклических чисел.

3. Если $\varepsilon^{3}=\varepsilon_{0}^{3}=0$, то $A l g_{3}^{\text {Vis }}\left(\mathbf{R} \mid 1, \varepsilon_{0}, \varepsilon_{0}^{2}\right):=\mathbf{R} 1+\mathbf{R} \varepsilon_{0}^{1}+\mathbf{R} \varepsilon_{0}^{2}=\left\{\mathcal{C}=r 1+g \varepsilon_{0}^{1}+b \varepsilon_{0}^{2} \mid\right.$ $r, g, b \in \mathbf{R}\}-$ цветная алгебра цветных нильпотентных чисел.

Цветные циклические числа формы $\mathcal{C}=x 1+y \varepsilon+z \varepsilon^{2}\left(\varepsilon^{3}=1\right)$ были впервые открыты Ч. Гревсом в работе [11]. Он назвал эти числа триплетами. Учитывая контекст данной 
работы, будем называть их ияетными числами. Сложение и умножение двух цветных чисел $\mathcal{C}_{1}=\left(r_{1}+g_{1} \varepsilon+b_{1} \varepsilon^{2}\right)$ и $\mathcal{C}_{2}=\left(r_{2}+g_{2} \varepsilon+b_{2} \varepsilon^{2}\right)$ определяются так:

$$
\begin{gathered}
\mathcal{C}_{1}+\mathcal{C}_{2}=\left(r_{1}+g_{1} \varepsilon+b_{1} \varepsilon^{2}\right)+\left(r_{2}+g_{2} \varepsilon+b_{2} \varepsilon^{2}\right)=\left(r_{1}+r_{2}\right)+\left(g_{1}+g_{2}\right) \varepsilon+\left(b_{1}+b_{2}\right) \varepsilon^{2}, \\
\mathcal{C}_{1} \cdot \mathcal{C}_{2}=\left(r_{1}+g_{1} \varepsilon+b_{1} \varepsilon^{2}\right)\left(r_{2}+g_{2} \varepsilon+b_{2} \varepsilon^{2}\right) \\
=\left(r_{1} r_{2}+g_{1} b_{2}+b_{1} g_{2}\right)+\left(r_{1} g_{2}+r_{2} g_{1}+b_{1} b_{2}\right) \varepsilon+\left(r_{1} b_{2}+g_{1} g_{2}+r_{2} b_{1}\right) \varepsilon^{2} .
\end{gathered}
$$

Легко проверить, что триплетное произведение изоморфно циклической свертке

$$
\begin{gathered}
\mathcal{C}_{1} \mathcal{C}_{2}=\left(r_{1}+g_{1} \varepsilon+b_{1} \varepsilon^{2}\right)\left(r_{2}+g_{2} \varepsilon+b_{2} \varepsilon^{2}\right) \equiv\left(r_{1}, g_{1}, b_{1}\right) *\left(r_{2}, g_{2}, b_{2}\right) \\
=\left(r_{1} r_{2}+g_{1} b_{2}+b_{1} g_{2}, r_{1} g_{2}+r_{2} g_{1}+b_{1} b_{2}, r_{1} b_{2}+g_{1} g_{2}+r_{2} b_{1}\right) .
\end{gathered}
$$

Триплетное сопряжение числа $\mathcal{C}=\left(r+g \varepsilon+b \varepsilon^{2}\right)$ описывается равенством

$$
\overline{\mathcal{C}}=\overline{r+g \varepsilon+b \varepsilon^{2}}=r+g \varepsilon^{2}+b \varepsilon^{1} .
$$

Норма $\|\mathcal{C}\|_{2}$ и модуль $|\mathcal{C}|_{2}$ определяются выражениями

$$
\|\mathcal{C}\|_{2}=\mathcal{C} \overline{\mathcal{C}}=\left(r^{2}+g^{2}+b^{2}\right)-(r g+r b+g b), \quad|\mathcal{C}|_{2}=\sqrt{\|\mathcal{C}\|_{2}} \equiv \sqrt{\mathcal{C} \overline{\mathcal{C}}}
$$

Ч. Гревс (см. [11]) показал, что каждое триплетное число имеет три нормы:

$$
\begin{gathered}
\|\mathcal{C}\|_{1}=|r+g+b|, \quad\|\mathcal{C}\|_{2}=\left(r^{2}+g^{2}+b^{2}\right)-(r g+r b+g b), \\
\|\mathcal{C}\|_{3}=\|\mathcal{C}\|_{1}\|\mathcal{C}\|_{2} \equiv r^{3}+g^{3}+b^{3}-3 r g b .
\end{gathered}
$$

Если расстояние $\rho(\mathcal{C}, \mathcal{D})$ между двумя триплетными числами $\mathcal{C}$ и $\mathcal{D}$ определить как модуль их разности $\mathcal{C}-\mathcal{D}=\mathcal{U}=r+g \varepsilon+b \varepsilon^{2}$, то в цветовом перцептуальном пространстве можно ввести три метрики:

$$
\begin{gathered}
\rho_{1}(\mathcal{C}, \mathcal{D})=|\mathcal{C}-\mathcal{D}|_{1}=|\mathcal{U}|_{1}=|r+g+b|, \\
\rho_{2}(\mathcal{C}, \mathcal{D})=|\mathcal{C}-\mathcal{D}|_{2}=|\mathcal{U}|_{2}=\sqrt[2]{\left(r^{2}+g^{2}+b^{2}\right)-(r g+r b+g b)}, \\
\rho_{3}(\mathcal{C}, \mathcal{D})=|\mathcal{C}-\mathcal{D}|_{3}=|\mathcal{U}|_{3}=\sqrt[3]{r^{3}+g^{3}+b^{3}-3 r g b}
\end{gathered}
$$

В результате чего алгебра цветных чисел $\mathcal{A} \lg _{3}^{V i s}\left(\mathbf{R} \mid 1, \varepsilon, \varepsilon^{2}\right)$ превратится в три 3-D метрических пространства:

$$
\begin{gathered}
\mathbf{G e o}_{3}^{V i s 1}=\left\langle\left\langle\mathrm{A}_{3}\left(\mathbf{R} \mid 1, \varepsilon, \varepsilon^{2}\right)|| r+g+b \mid\right\rangle\right\rangle, \\
\mathbf{G e o}_{3}^{V i s 2}=\left\langle\left\langle\mathrm{A}_{3}\left(\mathbf{R} \mid 1, \varepsilon, \varepsilon^{2}\right) \mid \sqrt[2]{\left(r^{2}+g^{2}+b^{2}\right)-(r g+r b+g b)}\right\rangle\right\rangle, \\
\mathbf{G e o}_{3}^{V i s 3}=\left\langle\left\langle\mathrm{A}_{3}\left(\mathbf{R} \mid 1, \varepsilon, \varepsilon^{2}\right) \mid \sqrt[3]{r^{3}+g^{3}+b^{3}-3 r g b}\right\rangle\right\rangle .
\end{gathered}
$$

Ч. Гревс дал алгебраическую и геометрическую интерпретации триплетным числам. С геометрической точки зрения цветное число $\mathcal{C}=x+y \varepsilon+z \varepsilon^{2}$ является точкой $\mathcal{C}=\mathcal{C}(x, y, z) \in \mathbf{V}_{R G B}^{3}$ в 3-D цветовом пространстве $\mathbf{V}_{R G B}^{3}$ с координатами $(x, y, z)$. C алгебраической точки зрения цветная алгебра является прямой суммой поля реальных чисел $\mathbf{R}$ и поля комплексных чисел $A l g_{3}^{V i s}=\mathbf{R} \cdot e_{l u}+\mathbf{C} \cdot \mathbf{E}_{c h}=\mathbf{R} \oplus \mathbf{C}$, где $\mathbf{e}_{l u}=\left(1+\varepsilon+\varepsilon^{2}\right) / 3, \mathbf{E}_{c h}=\left(1+\omega_{3} \varepsilon^{2}+\omega_{3}^{2} \varepsilon\right) / 3$ суть так называемые ортогональные идемпотенты (проекторы) $\mathbf{e}_{l u}^{2}=\mathbf{e}_{l u}, \mathbf{E}_{c h}^{2}=\mathbf{E}_{c h}, \mathbf{e}_{l u} \mathbf{E}_{c h}=\mathbf{E}_{c h} \mathbf{e}_{l u}=0$ и $\omega_{3}:=\exp (2 \pi / 3)$. Действительно, в соответствии с полиномиальной китайской теоремой об остатках имеем

$A l g_{3}^{V i s} \approx \mathbf{R}[x] /\left(x^{3}-1\right)=\mathbf{R}[x] /(x-1)\left(x^{2}+x+1\right) \approx \mathbf{R}[x] /(x-1) \oplus \mathbf{R}[x] /\left(x^{2}+x+1\right) \approx \mathbf{R} \oplus \mathbf{C}$. 
Следовательно, каждое цветное число $\mathcal{C}=x+y \varepsilon+z \varepsilon^{2}$ является линейной комбинацией $\mathcal{C}=$ $a_{l u} \cdot \mathbf{e}_{l u}+Z_{c h} \cdot \mathbf{E}_{c h}=\left(a_{l u}, Z_{c h}\right)$ реальной $a_{l u} \cdot \mathbf{e}_{l u}$ и комплексной $Z_{c h} \cdot \mathbf{E}_{c h}$ составляющих в идемпотентном базисе $\left\{\mathbf{e}_{l u}, \mathbf{E}_{c h}\right\}$, где

$$
\begin{gathered}
a_{l u} \cdot \mathbf{e}_{l u}=\mathcal{C} \cdot \mathbf{e}_{l u}=\mathbf{e}_{l u}=\left(r+g \varepsilon+b \varepsilon^{2}\right)\left[\left(1+\varepsilon+\varepsilon^{2}\right) / 3\right]=(r+g+b)\left[\left(1+\varepsilon+\varepsilon^{2}\right) / 3\right], \\
Z_{c h} \cdot \mathbf{E}_{c h}=\mathcal{C} \cdot \mathbf{E}_{c h}=\mathbf{e}_{l u}=\left(r+g \varepsilon+b \varepsilon^{2}\right)\left[\left(1+\omega_{3} \varepsilon^{2}+\omega_{3}^{2} \varepsilon\right) / 3\right]=\left(r+g \omega_{3}^{1}+b \omega_{3}^{2}\right)\left[\left(1+\omega_{3} \varepsilon^{2}+\omega_{3}^{2} \varepsilon\right) / 3\right]
\end{gathered}
$$

и поэтому

$$
a_{l u}=(r+g+b), \quad Z_{c h}=\left(r+g \omega_{3}^{1}+b \omega_{3}^{2}\right)=(r-(g+b) / 2)+i \sqrt{3}(g-b) / 2 .
$$

Будем называть $a_{l u} \in \mathbf{R}$ яркостными, а $Z_{c h} \in \mathbf{C}$ хроматическими числами. По этой причине цветные изображения можно рассматривать в двух форматах:

$$
\mathbf{f}(\mathbf{z})=f_{R}(\mathbf{z}) 1+f_{G}(\mathbf{z}) \varepsilon+f_{B}(\mathbf{z}) \varepsilon^{2}, \quad \mathbf{f}(\mathbf{z})=f_{l u}(\mathbf{z}) \mathbf{e}_{l u}+\mathbf{f}_{c h}(\mathbf{z}) \mathbf{E}_{c h}=\left(f_{l u}(\mathbf{z}), \mathbf{f}_{c h}(\mathbf{z})\right) .
$$

Первое представление называется ( $\mathrm{R}, \mathrm{G}, \mathrm{B})$-форматом, а второе - яркостно-хроматическим ("luminance-chrominance"-LC). Последний формат $\left(f_{l u}(\mathbf{z}), \mathbf{f}_{c h}(\mathbf{z})\right)$ определяет изображение в терминах яркостной $f_{l u}(\mathbf{z})$ и хроматической $\mathbf{f}_{c h}(\mathbf{z})$ составляющих, где $\left|\mathbf{f}_{c h}(\mathbf{z})\right|$ - насыщенность и $\arg \left\{\mathbf{f}_{c h}(\mathbf{z})\right\}$ - цветовой тон $\mathbf{f}(\mathbf{z})$.

Изменения цветового тона, насыщенности и яркости достаточно просто описываются на языке цветной алгебры. Пусть для примера $\mathrm{A}=\left(a_{l u}, Z_{c h}\right)=\left(a_{l u},\left|Z_{c h}\right| e^{i \varphi}\right)$, где $a_{l u}>0$, тогда преобразования

$$
\begin{gathered}
\mathbf{f}(\mathbf{z}) \rightarrow \mathrm{A} \cdot \mathbf{f}(\mathbf{z}) \\
=\left(a_{l u}, Z_{c h}\right)\left(f_{l u}(\mathbf{z}), \mathbf{f}_{c h}(\mathbf{z})\right)=\left(a_{l u},\left|Z_{c h}\right| e^{i \varphi}\right)\left(f_{l u}(\mathbf{z}), \mathbf{f}_{c h}(\mathbf{z})\right)=\left(a_{l u} f_{l u}(\mathbf{z}),\left|Z_{c h}\right| e^{i \varphi} \mathbf{f}_{c h}(\mathbf{z})\right)
\end{gathered}
$$

меняют яркость, цветовой тон и насыщенность цветного изображения. Множество таких преобразований формирует яркостно-хроматическую группу

$$
\mathbf{L C G}\left(A l g_{3}^{V i s}(\mathbf{R} \mid \varepsilon)\right)=\left\{\left(a_{l u}, Z_{c h}\right) \mid\left(a_{l u} \in \mathbf{R}^{+}\right) \&\left(Z_{c h} \in \mathbf{C}\right)\right\} .
$$

В частности,

- если $\mathrm{A}=\left(a_{l u}, Z_{c h}\right)=\left(1, e^{i \varphi}\right)$, то преобразования

$$
\mathbf{f}(\mathbf{z}) \rightarrow \mathrm{A} \cdot \mathbf{f}(\mathbf{z})=\left(1, e^{i \varphi}\right)\left(f_{l u}(\mathbf{z}), \mathbf{f}_{c h}(\mathbf{z})\right)=\left(f_{l u}(\mathbf{z}), e^{i \varphi} \mathbf{f}_{c h}(\mathbf{z})\right)
$$

изменяют цветовой тон изображения. Множество подобных преобразований формирует ортогональную группу преобразований цветового тона $\mathbf{H O G}\left(\operatorname{Alg}_{3}^{V i s}(\mathbf{R} \mid \varepsilon)\right)=\left\{\left(1, e^{i \varphi}\right) \mid e^{i \varphi} \in \mathbf{C}\right\}$ (hue orthogonal group);

- пусть $\mathrm{A}=(1, s), s>0$, тогда преобразования

$$
\mathbf{f}(\mathbf{z}) \rightarrow \mathrm{A} \cdot \mathbf{f}(\mathbf{z})=(1, s)\left(f_{l u}(\mathbf{z}), \mathbf{f}_{c h}(\mathbf{z})\right)=\left(f_{l u}(\mathbf{z}), s \mathbf{f}_{c h}(\mathbf{z})\right)
$$

изменяют насыщенность исходного изображения. Множество таких преобразований формирует группу преобразований насыщенности $\mathbf{S a G}\left(A l g_{3}^{V i s}\left(\mathbf{R} \mid \varepsilon_{c o l}\right)\right)=\left\{(1, s) \mid s \in \mathbf{R}^{+}\right\}$(saturation group);

- если же $\mathrm{A}=\left(1, Z_{c h}\right)=\left(1, s e^{i \varphi}\right)$, то преобразования

$$
\mathbf{f}(\mathbf{z}) \rightarrow \mathrm{A} \cdot \mathbf{f}(\mathbf{z})=\left(1, s e^{i \varphi}\right)\left(f_{l u}(\mathbf{z}), \mathbf{f}_{c h}(\mathbf{z})\right)=\left(f_{l u}(\mathbf{z}), s e^{i \varphi} \mathbf{f}_{c h}(\mathbf{z})\right)
$$

изменяют цветовой тон и насыщенность исходного изображения. Множество таких преобразований формирует хроматическую группу

$$
\mathbf{C h G}\left(A l g_{3}^{V i s}\left(\mathbf{R} \mid \varepsilon_{c o l}\right)\right)=\left\{\left(1, s e^{i \varphi}\right) \mid\left(e^{i \varphi} \in \mathbf{C}\right) \&\left(s \in \mathbf{R}^{+}\right)\right\} .
$$




\section{3. Мультиплетные модели многоканальных изображений}

Традиционно многоканальные изображения интерпретируются как $K$-D векторно-значные сигналы $\mathbf{f}(\mathbf{x})=\left(f_{0}(\mathbf{x}), f_{1}(\mathbf{x}), \ldots, f_{K-1}(\mathbf{x})\right): \mathbf{R}^{n} \rightarrow \mathbf{V}^{K}$. Будем интерпретировать их как мультиплетно-значные сигналы $\mathbf{f}(\mathbf{x})=f_{0}(\mathbf{x})+f_{1}(\mathbf{x}) \varepsilon^{1}+f_{2}(\mathbf{x}) \varepsilon^{2}+\ldots+f_{K-1}(\mathbf{x}) \varepsilon^{K-1}$, которые принимают значения в мультиплетной алгебре $\operatorname{Alg}_{K}^{V i s}\left(\mathbf{R} \mid 1, \varepsilon, \ldots, \varepsilon^{K-1}\right)=\mathbf{R} 1+\mathbf{R} \varepsilon^{1}+\ldots+\mathbf{R} \varepsilon^{K-1}$, где $\mathbf{x} \in \mathbf{R}^{n}$ и $1, \varepsilon^{1}, \ldots, \varepsilon^{K-1}\left(\varepsilon^{K}=+1,0,-1\right)$ - мультицветные гипермнимые единицы, удовлетворяющие одному из следующих свойств $\varepsilon^{K}=+1,0,-1$ (см. [12-16]).

Любое мультиплетное число представляется линейной комбинацией гипермнимых единиц $\mathrm{M}=\sum_{i=0}^{K-1} a_{i} \varepsilon^{i}, a_{i} \in \mathbf{R}$. Эти числа (в зависимости от $\left.\varepsilon^{K}=+1,0,-1\right)$ формируют три мультиплетные алгебры:

$$
A l g_{K}^{+, V i s}(\mathbf{R}) \equiv \sum_{i=0}^{K-1} \mathbf{R} \varepsilon_{+}^{i}, \quad A l g_{K}^{-, V i s}(\mathbf{R}) \equiv \sum_{i=0}^{K-1} \mathbf{R} \varepsilon_{-}^{i}, \quad A l g_{K}^{0, V i s}(\mathbf{R}) \equiv \sum_{i=0}^{K-1} \mathbf{R} \varepsilon_{0}^{i} .
$$

Сложение мультиплетных чисел $\mathrm{M}_{1}$ и $\mathrm{M}_{2}$ в трех алгебрах осуществляется покомпонентно:

$$
\mathrm{M}=\mathrm{M}_{1}+\mathrm{M}_{2}=\sum_{i=0}^{K-1} a_{i} \varepsilon^{i}+\sum_{i=0}^{K-1} b_{i} \varepsilon^{i}=\sum_{i=0}^{K-1}\left(a_{i}+b_{i}\right) \varepsilon^{i} .
$$

Следовательно, по отношению к сложению все три алгебры формируют одно и то же векторное пространство. Правила умножения любой пары мультиплетных чисел $\mathrm{M}_{1}$ и $\mathrm{M}_{2}$ в трех мультиплетных алгебрах различны:

$$
\begin{gathered}
\mathrm{M}=\mathrm{M}_{1} \cdot \mathrm{M}_{2}=\left(\sum_{n=0}^{K-1} a_{n} \varepsilon_{+}^{n}\right)\left(\sum_{m=0}^{K-1} b_{m} \varepsilon_{+}^{m}\right)=\sum_{l=0}^{K-1}\left(\sum_{m=0}^{K-1} a_{l \ominus m} b_{m}\right) \varepsilon_{+}^{l}=\sum_{l=0}^{K-1} c_{l} \varepsilon_{+}^{l} \quad \text { для } \quad A l g_{K}^{+, V i s}(\mathbf{R}) ; \\
\mathrm{M}=\mathrm{M}_{1} \cdot \mathrm{M}_{2}=\left(\sum_{n=0}^{K-1} a_{n} \varepsilon_{-}^{n}\right)\left(\sum_{m=0}^{K-1} b_{m} \varepsilon_{-}^{m}\right)=\sum_{l=0}^{K-1}\left(\sum_{m=0}^{K-1} \operatorname{Sign}(l-m) a_{l \ominus m} b_{m}\right) \varepsilon_{-}^{l} \\
=\sum_{l=0}^{K-1} c_{l} \varepsilon_{-}^{l} \quad \text { для } \quad A l g_{K}^{-, V i s}(\mathbf{R}) ; \\
\mathrm{M}^{=} \mathrm{M}_{1} \cdot \mathrm{M}_{2}=\left(\sum_{n=0}^{K-1} a_{n} \varepsilon_{0}^{n}\right)\left(\sum_{m=0}^{K-1} b_{m} \varepsilon_{0}^{m}\right)=\sum_{l=0}^{K-1}\left(\sum_{m=0}^{K-1} \operatorname{Hev}(l-m) a_{l \ominus m} b_{m}\right) \varepsilon_{0}^{l} \\
=\sum_{l=0}^{K-1} c_{l} \varepsilon_{0}^{l} \quad \text { для } \quad \operatorname{Alg}_{K}^{0, V i s}(\mathbf{R}) .
\end{gathered}
$$

Легко видеть, что мультиплетные произведения изоморфны дискретным К-точечным сверткам - циклической, ациклической и нильпотентной соответственно:

$$
c_{l}=\sum_{m=0}^{K-1} a_{l \ominus m} b_{m}, \quad c_{l}=\sum_{m=0}^{K-1} \operatorname{Sign}(l-m) a_{l \ominus m} b_{m}, \quad c_{l}=\sum_{m=0}^{K-1} \operatorname{Hev}(l-m) a_{l \ominus m} b_{m},
$$

где $l \ominus m$ есть вычитание по модулю $K$. Используя полиномиальную китайскую теорему об остатках, можно легко доказать, что две алгебры, $A l g_{K}^{+, V i s}(\mathbf{R})$ и $A l g_{K}^{-, V i s}(\mathbf{R})$, являются прямыми суммами полей реальных и комплексных чисел:

$$
A l g_{K}^{+, V i s}(\mathbf{R}) \equiv\left(\mathbf{R} \cdot \mathbf{e}_{l u}^{1}\right) \oplus\left(\mathbf{R} \cdot \mathbf{e}_{l u}^{2}\right) \oplus \sum_{j=1}^{K / 2-1} \mathbf{C} \cdot \mathbf{E}_{c h}^{j}, \quad \text { если } \quad K-\text { четное }
$$


и

$$
A l g_{K}^{+, V i s}(\mathbf{R}) \equiv \mathbf{R}^{K_{l u}} \oplus \mathbf{C}^{K_{c h}}=\mathbf{R} \cdot \mathbf{e}_{l u}^{1} \oplus \sum_{j=1}^{(K-1) / 2} \mathbf{C} \cdot \mathbf{E}_{c h}^{j}, \quad \text { если } \quad K-\text { нечетное; }
$$

аналогично

$$
A l g_{K}^{-, V i s}(\mathbf{R})=\sum_{j=1}^{K / 2} \mathbf{C} \cdot \mathbf{E}_{c h}^{j}, \quad \text { если } \quad K \text { - четное }
$$

и

$$
A l g_{K}^{-, V i s}(\mathbf{R})=\mathbf{R} \cdot \mathbf{e}_{l u}^{1}+\sum_{j=1}^{(K-1) / 2} \mathbf{C} \cdot \mathbf{E}_{c h}^{j}, \quad \text { если } \quad K-\text { нечетное }
$$

где $\mathbf{e}_{l u}^{i}$ и $\mathbf{E}_{c h}^{j}$ — "реальные" и "комплексные" ортогональные идемпотенты, такие что $\left(\mathbf{e}_{l u}^{i}\right)^{2}=$ $\mathbf{e}_{l u}^{i},\left(\mathbf{E}_{c h}^{j}\right)^{2}=\mathbf{E}_{c h}^{j}, \mathbf{e}_{l u}^{i} \mathbf{E}_{c h}^{j}=\mathbf{E}_{c h}^{j} \mathbf{e}_{l u}^{i}$ для всех $i$ и $j$.

Пусть $K_{l u}=0,1,2$ и $K_{c h}=K / 2, K / 2-1,(K-1) / 2$. Каждый мультиплет $\mathrm{M} \in A l g_{K}^{ \pm, V i s}(\mathbf{R})$ может быть представлен в виде линейной комбинации $K_{l u}$ "скалярных" и $K_{c h}$ "комплексных" составляющих

$$
\mathrm{M}=\sum_{i=1}^{K_{l u}}\left(a_{i} \cdot \mathbf{e}_{l u}^{i}\right)+\sum_{j=1}^{K_{c h}}\left(\mathbf{z}_{j} \cdot \mathbf{E}_{c h}^{j}\right)
$$

Реальные числа $a_{i} \in \mathbf{R}$ называются мульти-яркостяли, а комплексные $\mathbf{z}_{j} \in \mathbf{C}-$ мультихроматами. В таком представлении две главные арифметические операции имеют простую форму:

$$
\begin{aligned}
& \mathrm{M}_{1}+\mathrm{M}_{2}=\left(\sum_{i=1}^{K_{l u}} a_{i} \cdot \mathbf{e}_{l u}^{i}+\sum_{j=1}^{K_{c h}} \mathbf{z}_{j} \cdot \mathbf{E}_{c h}^{j}\right)+\left(\sum_{i=1}^{K_{l u}} b_{i} \cdot \mathbf{e}_{l u}^{i}+\sum_{j=1}^{K_{c h}} \mathbf{w}_{j} \cdot \mathbf{E}_{c h}^{j}\right)=\left(\sum_{i=1}^{K_{l u}}\left(a_{i}+b_{i}\right) \cdot \mathbf{e}_{l u}^{i}+\sum_{j=1}^{K_{c h}}\left(\mathbf{z}_{j}+\mathbf{w}_{j}\right) \cdot \mathbf{E}_{c h}^{j}\right), \\
& \mathrm{M}_{1} \cdot \mathrm{M}_{2}=\left(\sum_{i=1}^{K_{l u}} a_{i} \cdot \mathbf{e}_{l u}^{i}+\sum_{j=1}^{K_{c h}} \mathbf{z}_{j} \cdot \mathbf{E}_{c h}^{j}\right) \cdot\left(\sum_{i=1}^{K_{l u}} b_{i} \cdot \mathbf{e}_{l u}^{i}+\sum_{j=1}^{K_{c h}} \mathbf{w}_{j} \cdot \mathbf{E}_{c h}^{j}\right)=\left(\sum_{i=1}^{K_{l u}}\left(a_{i} \cdot b_{i}\right) \cdot \mathbf{e}_{l u}^{i}+\sum_{j=1}^{K_{c h}}\left(\mathbf{z}_{j} \cdot \mathbf{w}_{j}\right) \cdot \mathbf{E}_{c h}^{j}\right) .
\end{aligned}
$$

Мультиплетные алгебры не являются полями. Они формируют числовые кольца с делителями нуля.

О п р е д е л е н и е 3. Многоканальные 2-D сигналы типа

$$
\mathbf{f}(\mathbf{z})=\sum_{i=0}^{K-1} f_{i}(\mathbf{z}) \varepsilon^{i}, \quad \mathbf{f}(\mathbf{z})=\sum_{i=1}^{K_{l u}}\left[f_{l u}^{i}(\mathbf{z}) \cdot \mathbf{e}_{l u}^{i}\right]+\sum_{j=1}^{K_{c h}}\left[\mathbf{f}_{c h}^{j}(\mathbf{z}) \cdot \mathbf{E}_{c h}^{j}\right]
$$

называются мультиплетно-значными изображениями в мультиплетном и мульти-яркостнохроматическом форматах соответственно.

Первый формат определяет изображение $K$ яркостями каждого канала. Второй формат определяет его $K_{l u}$ яркостными компонентами $\left(f_{l u}^{1}(\mathbf{z}), f_{l u}^{2}(\mathbf{z}), \ldots, f_{l u}^{K_{l u}}(\mathbf{z})\right)$ и $K_{c h}$ хроматическими компонентами $\left(\mathbf{f}_{c h}^{1}(\mathbf{z}), \mathbf{f}_{c h}^{2}(\mathbf{z}), \ldots, \mathbf{f}_{c h}^{K_{c h}}(\mathbf{z})\right)$, где $\left|\mathbf{f}_{c h}^{1}(\mathbf{z})\right|,\left|\mathbf{f}_{c h}^{2}(\mathbf{z})\right|, \ldots,\left|\mathbf{f}_{c h}^{K_{c h}}(\mathbf{z})\right|$ - мульти-насыщенности и $\arg \left\{\mathbf{f}_{c h}^{1}(\mathbf{z})\right\}, \arg \left\{\mathbf{f}_{c h}^{2}(\mathbf{z})\right\}, \ldots, \arg \left\{\mathbf{f}_{c h}^{K_{c h}}(\mathbf{x})\right\}$ - мульти-тона многоканального изображения $\mathbf{f}(\mathbf{z})$.

Изменения мульти-яркости и мульти-хроматичности у многоканального изображения легко описываются на языке мультиплетной алгебры $A l g V_{K}^{V i s}(\mathbf{R})$ как преобразование $\mathbf{f}(\mathbf{z}) \rightarrow \mathrm{M} \cdot \mathbf{f}(\mathbf{z})$ для подходящего мультиплетного числа М. Например, если

$$
\begin{gathered}
\mathrm{M}=\left(a_{l u}^{1}, a_{l u}^{2}, \ldots, a_{l u}^{K_{l u}} ; Z_{c h}^{1}, Z_{c h}^{2}, \ldots, Z_{c h}^{K_{c h}}\right) \\
=\left(a_{l u}^{1}, a_{l u}^{2}, \ldots, a_{l u}^{K_{l u}} ;\left|Z_{c h}^{1}\right| e^{i \varphi_{c h}^{1}},\left|Z_{c h}^{2}\right| e^{i \varphi_{c h}^{2}}, \ldots,\left|Z_{c h}^{K_{c h}}\right| e^{i \varphi_{c h}^{K_{c h}}}\right),
\end{gathered}
$$


то преобразование

$$
\begin{gathered}
\mathbf{f}(\mathbf{z}) \rightarrow \mathrm{M} \cdot \mathbf{f}(\mathbf{z})=\left(\sum_{i=1}^{K_{l u}}\left[a_{l u}^{i} \cdot \mathbf{e}_{l u}^{i}\right]+\sum_{j=1}^{K_{c h}}\left[\left|Z_{c h}^{j}\right| e^{i \varphi_{c h}^{j}} \cdot \mathbf{E}_{c h}^{j}\right]\right) \cdot\left(\sum_{i=1}^{K_{l u}}\left[f_{l u}^{i}(\mathbf{z}) \cdot \mathbf{e}_{l u}^{i}\right]+\sum_{j=1}^{K_{c h}}\left[\mathbf{f}_{c h}^{j}(\mathbf{z}) \cdot \mathbf{E}_{c h}^{j}\right]\right) \\
=\left(\sum_{i=1}^{K_{l u}}\left[a_{l u}^{i} f_{l u}^{i}(\mathbf{z}) \cdot \mathbf{e}_{l u}^{i}\right]+\sum_{j=1}^{K_{c h}}\left[\left|Z_{c h}^{j}\right| e^{i \varphi_{c h}^{j}} \mathbf{f}_{c h}^{j}(\mathbf{z}) \cdot \mathbf{E}_{c h}^{j}\right]\right)
\end{gathered}
$$

изменяет мульти-яркости, мульти-цветовые тона и мульти-насыщенности. Множество таких преобразований формирует группу

$$
\begin{gathered}
\operatorname{MLCG}\left(\mathrm{A}_{k}\left(\mathbf{R} \mid 1, \varepsilon^{1}, \varepsilon^{2}, \ldots, \varepsilon^{K-1}\right)\right) \\
=\left\{\left(a_{l u}^{1}, a_{l u}^{2}, \ldots, a_{l u}^{K_{l u}} ; Z_{c h}^{1}, Z_{c h}^{2}, \ldots, Z_{c h}^{K_{c h}}\right) \mid\left(a_{l u}^{1}, a_{l u}^{2}, \ldots, a_{l u}^{K_{l u}} \in \mathbf{R}^{+}\right) \&\left(Z_{c h}^{1}, Z_{c h}^{2}, \ldots, Z_{c h}^{K_{c h}} \in \mathbf{C}\right)\right\} .
\end{gathered}
$$

Мы предполагаем, что головной мозг может использовать гиперкомплексные алгебры для ментального изменения мульти-яркости и мульти-хроматичности многоканальных изображений, которые возникают в памяти мозга на так называемом "экране сознания", например во время сна.

\section{4. Синтез цветных преобразований, всплесков и сплайнов}

\section{1. Орто-унитарные преобразования цветных изображений}

Классический спектральный анализ, основанный на ортогональных и унитарных преобразованиях, играет важнейшую роль в цифровой обработке изображений. Преобразования, подобные дискретному преобразованию Фурье (ДПФ) и Уолша (ДПУ), интенсивно используются в различных приложениях (фильтрации, сжатии, оценке спектральной плотности и так далее). Естественным образом возникает вопрос о синтезе преобразований цветных (триплетнозначных) изображений. В этой части мы предлагаем широкий класс так называемых ортоунитарных преобразований для обработки цветных изображений.

Дискретные 2-D цветные $(N \times N)$-изображения $\mathbf{f}_{c o l}:=\left[\mathbf{f}_{c o l}(i, j)\right]_{i, j=1}^{N}$ определяются как 2-D $(N \times N)$-массивы с пикселями, записанными в $(\mathrm{R}, \mathrm{G}, \mathrm{B})$ или LC-форматах. Здесь каждый цветной пиксель $\mathbf{f}_{c o l}(i, j)$ в позиции $(i, j)$ является триплетным числом, записанным в $(\mathrm{R}, \mathrm{G}, \mathrm{B})$ или в LC форматах соответственно. Все изображения $\mathbf{f}_{c o l}:=\left[\mathbf{f}_{c o l}(i, j)\right]_{i, j=1}^{N}$ формируют $N^{2}$-мерное векторное пространство $\left(A l g_{3}^{V i s}\right)^{N^{2}}$ над триплетной алгеброй.

О п р е д е л е н и е 4 . Величина $\mathrm{N}_{k}\left(\mathbf{f}_{c o l}\right):=\sum_{(i, j) \in \mathbf{Z}_{N}^{2}}\left\|\mathbf{f}_{c o l}(i, j)\right\|_{k}$ называется нормой изображения $\mathbf{f}_{\text {col }}$, где $\|\cdot\|_{k}, k=1,2,3$, - одна из трех триплетных норм (3) .

О п р е д е л е н и е 5. Линейный оператор $\mathrm{L}_{2 D}:\left(A l g_{3}^{V i s}\right)^{N^{2}} \rightarrow\left(A l g_{3}^{V i s}\right)^{N^{2}}$ называется орто-унитарным (или цветным), если он сохраняет норму цветного изображения.

Заметим, что ортогональные изображения сохраняют норму у реально-значных (серых) изображений, а унитарные - у комплексно-значных (бихроматических) изображений. Поскольку триплетное число в LC-формате является своеобразным агрегатом из действительного и комплексного чисел, то естественно назвать введенные преобразования орто-унитарными или цветными. B LC-формате такие преобразования могут быть синтезированы с помощью произвольного ортогонального $O_{2 D}$ и унитарного $U_{2 D} 2-\mathrm{D}$ преобразований следующим образом:

$$
\mathrm{L}_{2 D}=O_{2 D} \mathbf{e}_{l u}+U_{2 D} \mathbf{E}_{c h}=\left(O_{1 D} \otimes O_{1 D}\right) \mathbf{e}_{l u}+\left(U_{1 D} \otimes U_{1 D}\right) \mathbf{E}_{c h},
$$

где $\otimes-$ символ тензорного произведения. Следовательно, каждая пара $\left(O_{1 D}, U_{1 D}\right)$ из ортогонального $O_{1 D}$ и унитарного $U_{1 D}$ преобразований генерирует орто-унитарное преобразование $\mathrm{L}_{2 D}=\left(O_{1 D} \otimes O_{1 D}\right) \mathbf{e}_{l u}+\left(U_{1 D} \otimes U_{1 D}\right) \mathbf{E}_{c h}$.

В качестве примера ниже приведены некоторые цветные преобразования. 


\begin{tabular}{|l|l|l|l|l|}
\hline & $F$ & $W$ & $\dot{H} d$ & $\dot{W}$ \\
\hline$W$ & $W \cdot \mathbf{e}_{l u}+F \cdot \mathbf{E}_{c h}$ & $W \cdot \mathbf{e}_{l u}+W \cdot \mathbf{E}_{c h}$ & $W \cdot \mathbf{e}_{l u}+\dot{H} d \cdot \mathbf{E}_{c h}$ & $W \cdot \mathbf{e}_{l u}+\dot{W v} \cdot \mathbf{E}_{c h}$ \\
\hline$H d$ & $H d \cdot \mathbf{e}_{l u}+F \cdot \mathbf{E}_{c h}$ & $H d \cdot \mathbf{e}_{l u}+W \cdot \mathbf{E}_{c h}$ & $H d \cdot \mathbf{e}_{l u}+H d \cdot \mathbf{E}_{c h}$ & $H d \cdot \mathbf{e}_{l u}+W v \cdot \mathbf{E}_{c h}$ \\
\hline$H t$ & $H t \cdot \mathbf{e}_{l u}+F \cdot \mathbf{E}_{c h}$ & $H t \cdot \mathbf{e}_{l u}+\dot{W} \cdot \mathbf{E}_{c h}$ & $H t \cdot \mathbf{e}_{l u}+\dot{H} d \cdot \mathbf{E}_{c h}$ & $H t \cdot \mathbf{e}_{l u}+\dot{W v} \cdot \mathbf{E}_{c h}$ \\
\hline$H r$ & $H r \cdot \mathbf{e}_{l u}+F \cdot \mathbf{E}_{c h}$ & $H r \cdot \mathbf{e}_{l u}+\dot{W} \cdot \mathbf{E}_{c h}$ & $H r \cdot \mathbf{e}_{l u}+\dot{H} d \cdot \mathbf{E}_{c h}$ & $H r \cdot \mathbf{e}_{l u}+\dot{W v} \cdot \mathbf{E}_{c h}$ \\
\hline$W v$ & $W v \cdot \mathbf{e}_{l u}+F \cdot \mathbf{E}_{c h}$ & $W v \cdot \mathbf{e}_{l u}+\dot{W} \cdot \mathbf{E}_{c h}$ & $W v \cdot \mathbf{e}_{l u}+\dot{H} d \cdot \mathbf{E}_{c h}$ & $W v \cdot \mathbf{e}_{l u}+\dot{W v} \cdot \mathbf{E}_{c h}$ \\
\hline
\end{tabular}

Здесь $O_{1 D}=\mathrm{W}, \mathrm{Hd}, \mathrm{Ht}, \mathrm{Hr}, \mathrm{Wv}$ суть ортогональные преобразования Уолша, Адамара, Хартли, Хаара и вейвлет-преобразование соответственно; $U_{1 D}=F, \dot{W}, \dot{H} d, \dot{W} v-$ унитарное преобразование Фурье, комплексно-значное преобразование Уолша и Адамара и, наконец, комплексно-значное вейвлет-преобразование соответственно.

Если $O=\left[\varphi_{k}(n)\right]_{k, n=0}^{N-1}$ и $U=\left[\psi_{k}(n)\right]_{k, n=0}^{N-1}-$ произвольные ортогональное и унитарные преобразования, строки которых образуют базисы из реально- и комплексно-значных функций $\left\{\varphi_{k}(n)\right\}_{k, n=0}^{N-1}$ и $\left\{\psi_{k}(n)\right\}_{k, n=0}^{N-1}$, то выражение

$$
\begin{gathered}
(O \otimes O) \mathbf{e}_{l u}+(U \otimes U) \mathbf{E}_{c h}=\left(\left[\varphi_{k_{1}}\left(n_{1}\right)\right] \otimes\left[\varphi_{k_{2}}\left(n_{2}\right)\right]\right) \mathbf{e}_{l u}+\left[\psi_{k_{1}}\left(n_{1}\right)\right] \otimes\left[\psi_{k_{2}}\left(n_{2}\right)\right] \mathbf{E}_{c h} \\
=\left[\varphi_{k_{1}}\left(n_{1}\right) \varphi_{k_{2}}\left(n_{2}\right)\right] \mathbf{e}_{l u}+\left[\psi_{k_{1}}\left(n_{1}\right) \psi_{k_{2}}\left(n_{2}\right)\right] \mathbf{E}_{c h}
\end{gathered}
$$

представляет цветное преобразование, где

$$
\left\{\varphi_{k_{1}}\left(n_{1}\right) \varphi_{k_{2}}\left(n_{2}\right)\right\}_{k_{1}, k_{2}=0, n_{1}, n_{2}=0}^{N-1, N-1} \quad \text { и } \quad\left\{\psi_{k_{1}}\left(n_{1}\right) \psi_{k_{2}}\left(n_{2}\right)\right\}_{k_{1}, k_{2}=0, n_{1}, n_{2}=0}^{N-1, N-1}
$$

формируют $N^{2}$ ортогональных и унитарных базисных функций.

\section{2. Орто-унитарные (цветные) всплески}

Пусть $\psi^{R}(x)$ - реально-значная материнская вейвлет-функция (всплеск), а $\psi_{s, \tau}^{R}(x)$ - ее сдвинутые и масштабированные версии:

$$
\psi_{s, \tau}^{R}(x)=(\sqrt{|s|})^{-1} \psi^{R}\left(\frac{x-\tau}{s}\right), \quad s, \tau \in R, \quad s \neq 0 .
$$

Они формируют ортогональный базис пространства $L_{2}(\mathbf{R})$. Построим цветные всплески как агрегат из яркостной и хроматической составляющих. В качестве первой используем реальнозначный всплеск $\psi^{R}(x)$. Хроматическую составляющую определим как аналитический комплексно-значный сигнал вида

$$
\psi_{s, \tau}^{C h}(x)=\psi_{s, \tau}^{R}(x)+j \mathrm{H}_{1}\left\{\psi_{s, \tau}^{R}(x)\right\},
$$

где $\mathrm{H}_{1}\{\cdot\}$ - одномерное преобразование Гильберта. Используя идемпотенты $\mathbf{e}_{l u}, \mathbf{E}_{C h}$, построим цветные базисные всплески

$$
\begin{gathered}
\psi_{s, \tau}^{C o l}(x)=\varphi_{s, \tau}^{l u}(x) \cdot \mathbf{e}_{l u}+\psi_{s, \tau}^{C h}(x) \cdot \mathbf{E}_{C h}=\varphi_{s, \tau}^{l u}(x) \cdot \mathbf{e}_{l u}+\left[\varphi_{s, \tau}^{l u}(x)+j \mathrm{H}\left\{\varphi_{s, \tau}^{l u}(x)\right\}\right] \cdot \mathbf{E}_{C h} \\
=\varphi_{s, \tau}^{l u}(x) \cdot\left[\mathbf{e}_{l u}+\mathbf{E}_{C h}\right]+j \mathrm{H}\left\{\varphi_{s, \tau}^{l u}(x)\right\} \cdot \mathbf{E}_{C h}=\varphi_{s, \tau}^{l u}(x) \cdot \mathbf{I}_{3}+j \mathrm{H}\left\{\varphi_{s, \tau}^{l u}(x)\right\} \cdot \mathbf{E}_{C h},
\end{gathered}
$$

где $\varphi_{s, \tau}^{l u}(x)=\varphi_{s, \tau}^{R}(x)$ - реально-значная (яркостная) компонента всплеска; $\psi_{s, \tau}^{C h}(x)-$ его комплексно-значная (хроматическая) составляющая, $\mathbf{I}_{3}=\mathbf{e}_{l u}+\mathbf{E}_{C h}-$ единичное $(3 \times 3)$ преобразование. Очевидно, аналитические функции двух переменных

$$
\begin{aligned}
\psi_{s_{1}, \tau_{1}}^{C o l}(x) \psi_{s_{2}, \tau_{2}}^{C o l}(y)= & \varphi_{s_{1}, \tau_{1}}^{l u}(x) \varphi_{s_{2}, \tau_{2}}^{l u}(y) \cdot \mathbf{e}_{l u}+\left[\varphi_{s_{1}, \tau_{1}}^{l u}(x) \varphi_{s_{2}, \tau_{2}}^{l u}(y)+j \mathrm{H}_{2}\left\{\varphi_{s_{1}, \tau_{1}}^{l u}(x) \varphi_{s_{2}, \tau_{2}}^{l u}(y)\right\}\right] \cdot \mathbf{E}_{C h} \\
& =\varphi_{s_{1}, \tau_{1}}^{l u}(x) \varphi_{s_{2}, \tau_{2}}^{l u}(y) \cdot \mathbf{I}_{3}+j \mathrm{H}_{2}\left\{\varphi_{s_{1}, \tau_{1}}^{l u}(x) \varphi_{s_{2}, \tau_{2}}^{l u}(y)\right\} \cdot \mathbf{E}_{C h}
\end{aligned}
$$

формируют двумерные всплески, где $\mathrm{H}_{2}\{\cdot\}$ - двумерное преобразование Гильберта.

Мы определяем двумерное цветное (орто-унитарное) вейвлет-преобразование как

$$
F_{C O U T}^{c o l}\left(s_{1}, \tau_{1}, s_{2}, \tau_{2}\right)=\left(\sqrt{\left|s_{1}\right|\left|s_{2}\right|}\right)^{-1} \int_{-\infty}^{+\infty} \int_{-\infty}^{+\infty} \mathbf{f}_{c o l}(x, y) \psi_{s_{1}, \tau_{1}}^{C o l}(x) \psi_{s_{2}, \tau_{2}}^{C o l}(y) d x d y .
$$




\section{3. Орто-унитарные сплайны}

Подобно цветным всплескам можно построить цветные сплайны. Пусть $\operatorname{Spl}(x)-$ peальнозначный сплайн. Цветной сплайн есть триплетно-значная функция

$$
\begin{gathered}
S p l l^{C o l}(x)=S p l(x) \cdot \mathbf{e}_{l u}+[S p l(x)+j \mathrm{H}\{S p l(x)\}] \cdot \mathbf{E}_{C h} \\
=S p l(x) \cdot\left[\mathbf{e}_{l u}+\mathbf{E}_{C h}\right]+j \mathrm{H}\{S p l(x)\} \cdot \mathbf{E}_{C h}=S p l(x) \cdot \mathbf{I}_{3}+j \mathrm{H}\{S p(x)\} \cdot \mathbf{E}_{C h} .
\end{gathered}
$$

Пусть к примеру $S p l(x) \equiv B S p l(x)$ - произвольный В-сплайн, который строится с помощью итерационной свертки прямоугольного импульса

$$
B \operatorname{Spl}_{0}(x)=\left\{\begin{array}{ll}
1, & -1 / 2<x<1 / 2, \\
1 / 2, & |x|=1 / 2, \\
0 & \text { иначе },
\end{array} \quad \text { при } \quad B S p l_{n}(x)=\left(B S p l_{n-1} * B S p l_{0}\right)(x),\right.
$$

где * - символ свертки. В соответствии с (4) цветной одномерный В-сплайн имеет следующую форму:

$$
B S p l_{n}^{C o l}(x)=B S p l_{n}(x) \cdot \mathbf{I}_{3}+j \mathrm{H}\left\{B S p l_{n}(x)\right\} \cdot \mathbf{E}_{C h} .
$$

Очевидно, функции

$$
B S p l_{n}^{C o l}(x) \cdot B S p l_{n}^{C o l}(y)=B S p l_{n}(x) \cdot B S p l_{n}(y) \cdot \mathbf{I}_{3}+j \mathrm{H}_{2}\left\{B S p l_{n}(x) \cdot B S p l_{n}(y)\right\} \cdot \mathbf{E}_{C h}
$$

представляют двумерные сплайны.

\section{Заключение}

Разработан новый алгебраический подход к математическим моделям многоканальных изображений, основанный на коммутативных гиперкомплексных алгебрах. Цель работы состояла в том, чтобы показать, что гиперкомплексные алгебры являются адекватным математическим аппаратом для описания многоканальных изображений. Можно привести достаточное количество аргументов в пользу того, что мозг животных в процессе эволюции приобрел способность оперировать гиперкомплексными числами в процессе обработки и распознавания изображений. Поэтому мозг животных может рассматриваться как вычислительное устройство, работающее в некоторой гиперкомплексной алгебре.

\section{СПИСОК ЛИТЕРАТУРЫ}

1. Cronin T. A retina with at least ten spectral types of photoreceptors in a mantis shrimp // Nature. 1989. Vol. 339. P. 137-140. doi: 10.1038/339137a0 .

2. Chang C. Hyperspectral data processing: Algorithm design and analysis. N Y: Wiley Press, 2013. $1164 \mathrm{p}$.

3. Schowengerdt R. A. Remote sensing - Models and methods for image processing. N Y: Acad. Press, 1997. $558 \mathrm{p}$.

4. Soifer V. A. Computer image processing. Part II: Methods and algorithms. Berlin: VDM, Verlag, 2010. $584 \mathrm{p}$.

5. Luneburg R. K. The metric methods in binocular visual space// J. Opt. Soc. Amer. 1950. Vol. 40, no. 1. P. $627-642$.

6. Luneburg R. K. The metric methods in binocular visual // Studies and Essays. Courant Anniv. 1948. Vol. 11, № 1. P. 215-239.

7. Labunets V. Clifford algebra as unified language for image processing and pattern recognition // Computational noncommutative algebra and applications / eds. J. Byrnes, G. Ostheimer. Dordrect; Boston; London: Kluwer Acad. Publ., 2003. P. 197-225. doi: 10.1007/1-4020-2307-3_8.

8. Labunets V. G., Rundblad E. V., Astola J. Is the Brain a "Clifford algebra quantum computer"? Applied geometrical algebras in computer science and engineering / eds. L. Dorst, C. Doran, J. Lasenby. N Y: Birkhäuser, 2002. P. 486-495. doi: 978-1-4612-0089-5_25. 
9. Labunets V., Labunets-Rundblad E. V. Algebra and geometry of color images // Proc. of the First Int. Workshop on Spectral Tecniques and Logic Design for Future Digital Systems / eds. J. Astola, R. Stancovic, Tampere: Tampere University Publ., 2000. P. 231-261.

10. Doran C. J. L. Geometric algebra and its application to mathematical physics. Cambridge: Cambridge University Publ., 1994. 324 p.

11. Greaves Ch. On algebraic triplets // Proc. Irisn Acad. 1847. Vol. 3. P. 51-108.

12. Rundblad-Ostheimer E., Labunets V. Spatial-color Clifford algebras for invariant image recognition // Geometric computing with Clifford algebras / ed. G. Sommer. Berlin: Springer, 2001. P. 155-185. doi: 10.1007/978-3-662-04621-0_7.

13. Rundblad-Ostheimer E., Nikitin I., Labunets V. Unified approach to Fourier-Clifford-Prometheus sequences, transforms and filter banks // Computational Noncommutative Algebra and Applications / eds. J. Byrnes, G. Ostheimer. Dordrect; Boston; London: Kluwer Acad. Publ., 2003. P. 389-400.

doi: 10.1007/1-4020-2307-3_14.

14. Rundblad-Ostheimer E., Maidan E. A., Novak P., Labunets V. G. Fast color Haar-Prometheus wavelet transforms for image processing // Computational Noncommutative Algebra and Applications / ed. J. Byrnes, G. Ostheimer. Dordrect; Boston; London: Kluwer Acad. Publ., 2003. P. 389-400. doi: 10.1007/1-4020-2307-3_15.

15. Rundblad-Ostheimer E., Labunets V., Astola J. Is the visual cortex a "Fast Clifford algebra quantum computer"? // Clifford analysis and its applications / Mathematics, Physics and Chemistry. NATO Science Series. 2001. Vol. 25. P. 173-183. doi: 10.1007/978-94-010-0862-4_17.

16. Labunets V. G., Maidan A., Rundblad-Ostheimer E., Astola J. Colour triplet-valued wavelets and splines // Proc. of the 2nd International Symposium on Image and Signal Processing and Analysis. In conjunction with 23rd International Conference on Information Technology Interfaces (IEEE Cat. No.01EX480). Pula, 2001. P. 535-541. doi: 10.1109/ISPA.2001.938687.

Поступила 12.05.2020

После доработки 10.06.2020

Лабунец Валерий Григорьевич

Принята к публикации 6.07.2020

д-р техн. наук, профессор

Уральский государственный лесотехнический университет

г. Екатеринбург

e-mail: vlabunets05@yahoo.com

\section{REFERENCES}

1. Cronin T. A retina with at least ten spectral types of photoreceptors in a mantis shrimp. Nature, 1989, vol. 339, pp. 137-140. doi: 10.1038/339137a0 .

2. Chang C. Hyperspectral data processing: Algorithm design and analysis. N Y: Wiley Press, 2013, 1164 p.

3. Schowengerdt R. A. Remote sensing - Models and methods for image processing, N Y: Acad. Press, $1997,558 \mathrm{p}$.

4. Soifer V. A. Computer image processing. Part II: Methods and algorithms. Berlin: VDM, Verlag, 2010, $584 \mathrm{p}$.

5. Luneburg R. K. The metric methods in binocular visual space. J. Opt. Soc. Amer., 1950, vol. 40, no. 1, pp. $627-642$.

6. Luneburg R. K. The metric methods in binocular visual // Studies and Essays. Courant Anniv., 1948, vol. 11, no. 1, pp. 215-239.

7. Labunets V. Clifford algebra as unified language for image processing and pattern recognition. In: Computational Noncommutative Algebra and Applications, eds. J. Byrnes, G. Ostheimer. Dordrect; Boston; London: Kluwer Acad. Publ., 2003, pp. 197-225. doi: 10.1007/1-4020-2307-3_8.

8. Labunets V.G., Rundblad E. V., Astola J. Is the Brain a "Clifford algebra quantum computer"? In Applied Geometrical Algebras in Computer Science and Engineering, eds. L. Dorst, C. Doran, J. Lasenby, N Y: Birkhäuser, 2002, pp. 486-495. doi: 978-1-4612-0089-5_25.

9. Labunets V., Labunets-Rundblad E. V. Algebra and geometry of color images. In Proc. of the First Int. Workshop on Spectral Tecniques and Logic Design for Future Digital Systems, eds. J. Astola, R. Stancovic, Tampere: Tampere University Publ., 2000, pp. 231-261. 
10. Doran C. J.L. Geometric algebra and its application to mathematical physics, Cambridge: Cambridge University Publ., 1994, 324 p.

11. Greaves Ch. On algebraic triplets. Proc. Irisn Acad., 1847, vol. 3, pp. 51-108.

12. Rundblad-Ostheimer E., Labunets V. Spatial-color Clifford algebras for invariant image recognition. In Geometric Computing with Clifford Algebras, ed. G. Sommer, Berlin: Springer, 2001, pp. 155-185. doi: 10.1007/978-3-662-04621-0_7.

13. Rundblad-Ostheimer E., Nikitin I., Labunets V. Unified approach to Fourier-Clifford-Prometheus sequences, transforms and filter banks. In Computational Noncommutative Algebra and Applications, eds. J. Byrnes, G. Ostheimer. Dordrect, Boston, London: Kluwer Acad. Publ., 2003, pp. 389-400. doi: 10.1007/1-4020-2307-3_14.

14. Rundblad-Ostheimer E., Maidan E. A., Novak P., Labunets V. G. Fast color Haar-Prometheus wavelet transforms for image processing. In Computational Noncommutative Algebra and Applications, ed. J. Byrnes, G. Ostheimer. Dordrect, Boston, London, Kluwer Acad. Publ., 2003, pp. 389-400. doi: 10.1007/1-4020-2307-3_15.

15. Rundblad-Ostheimer E., Labunets V., Astola J. Is the visual cortex a "Fast Clifford algebra quantum computer"? In Clifford analysis and its applications / Mathematics, Physics and Chemistry. NATO Science Series, 2001, vol. 25, pp. 173-183. doi: 10.1007/978-94-010-0862-4_17.

16. Labunets V. G., Maidan A., Rundblad-Ostheimer E., Astola J. Colour triplet-valued wavelets and splines. Proc. of the 2nd International Symposium on Image and Signal Processing and Analysis. In conjunction with 23rd International Conference on Information Technology Interfaces (IEEE Cat. No.01EX480), Pula, 2001, pp. 535-541. doi: 10.1109/ISPA.2001.938687.

Received May 12, 2020

Revised June 10, 2020

Accepted July 6, 2020

Funding Agency: This work was supported by the Russian Foundation for Basic Research (project no. 19-29-09022\19.)

Valerii Grigor'evich Labunets, Dr. Techn. Sci., Prof., Ural State Forest Engineering University, Yekaterinburg, 620100 Russia, e-mail: vlabunets05@yahoo.com .

V. G. Labunets. Hypercomplex models of multichannel images, Trudy Instituta Matematiki i Mekhaniki URO RAN, 2020, vol. 26, no. 3, pp. 69-83. 